\title{
Mechanical response of fiber-reinforced sand under cyclic loading
}

\author{
Gang $\mathrm{Li}^{1}$, Jinli Zhang ${ }^{2, *}$, and Jia Liu ${ }^{3}$ \\ ${ }^{1}$ Shaanxi Key Laboratory of Safety and Durability of Concrete Structures, Xijing University, Xi'an, Shaanxi 710123, China; \\ ${ }^{2}$ State Key Laboratory of Coastal and Offshore Engineering, Dalian University of Technology, Dalian, Liaoning 116024, China; \\ ${ }^{3}$ School of Geological Engineering and Geomatics, Chang'an University, Xi'an, Shaanxi 710054, China.
}

\begin{abstract}
Based on the consolidated undrained test under cyclic loading, the mechanical response of fiber-reinforced sand under cyclic loading was studied by varying fiber content $(0.2 \%$ and $0.4 \%)$, fiber length $(7 \mathrm{~mm}$ and $13 \mathrm{~mm})$, and cyclic stress ratio $(0.22,0.24$ and 0.26$)$, and the effects of fiber content and fiber length on the excess pore water pressure, dynamic strength and dynamic shear modulus were discussed. The results showed that with the increasing of fiber content, the dynamic strength and liquefaction resistance increased, whereas the accumulation rate of excess pore water pressure decreased. The reduction rate of dynamic shear modulus decreased with the increasing of fiber length, and the dynamic strength decreased with the increasing of cyclic stress ratio, which indicated that the dynamic characteristics of fiber-reinforced sand were better than that of unreinforced sand.
\end{abstract}

\section{Introduction}

In recent years, the scale of traffic infrastructure continues to expand, and the requirements for subgrade fillers are higher and higher. Fiber-reinforced technology is a new method of geosynthetics by adding fiber or mesh into soil. Because the fiber is evenly distributed in the soil, it can play the role of reinforcement in all directions, so it can significantly improve the strength and deformation capacity of the soil, without causing environmental pollution, which has been widely concerned in engineering applications. Ghavami et al. [1] studied the physical and mechanical properties of coconut shell fiber and bamboo fiber, and found that natural fiber as reinforcement materials have achieved ideal results, which verified the feasibility of natural fiber to improve the mechanical performance of soil. Welker and Josten [2] carried out direct shear test on fiber-reinforced soil under the condition of optimal moisture content. By comparing the peak shear strength of soil sample, the optimal fiber content of soil is $0.2 \%$. Yetimoglu and Salbas [3] tested the shear strength of fiber-reinforced soil, and found that the addition of fiber had no significant effect on the peak value of shear strength of soil, but significantly increased the residual shear strength of soil. Consoli et al. [4] conducted triaxial compression test and field plate test on fiber-reinforced soil, and the test results showed that the addition of fiber could improve the soil. Especially in the case of large deformation, the reinforcement effect of fiber is more obvious. Mesbah et al. [5] carried out direct tensile test on fiber-reinforced soil to evaluate the tensile performance of the soil. The results showed that the fiber can inhibit the expansion of the initial crack to a certain extent. Kumar et al. [6] conducted compressive strength test and tensile strength test on fiber-reinforced soil and calculated the ratio of tensile strength to compressive strength of soil. It was found that with the increasing of fiber content, the increase of tensile strength was higher than that of compressive strength. Based on the compressive strength test, Consoli et al. [7] found that the compressive strength of cement soil can be significantly improved by adding appropriate amount of fiber. Akbulut et al. [8] studied the shear strength characteristics of fiber-reinforced clay, and found that adding fiber can increase the cohesion and internal friction angle of clay. Hamidi and hooersfand [9] noted that the stress-strain characteristics of polypropylene fiber reinforced soil showed typical strain hardening type. Tang et al. [10] pointed out that the reinforcement mechanism of fiber mainly depends on the interaction between fiber and clay.

At present, there are many researches on fiber-reinforced soil, but few researches on fiber reinforced sand, especially on the mechanical properties of fiber-reinforced sand under cyclic loading. According to control the relative density $\left(D_{\mathrm{r}}\right)$ and confining pressure $\left(\sigma_{3}\right)$, the dynamic triaxial tests were carried out to study the influence of fiber content $(\zeta)$ and fiber length $\left(l_{\mathrm{f}}\right)$ on the excess pore water pressure $(u)$, dynamic strength $\left(\sigma_{\mathrm{d}}\right)$ and dynamic shear modulus $\left(G_{\mathrm{N}}\right)$ of fiber-reinforced sand, which provide a reference for practical engineering application.

* Corresponding author: jlzhang@dlut.edu.cn 


\section{Materials and method}

\subsection{Test materials}

The sand used in the experiment is river sand from Dalian. After the sand is sieved for $1 \mathrm{~mm}$, remove the soil sample and wash it with water, and then pass the 0.075 $\mathrm{mm}$ sieve to remove the silt and clay in the sand. After drying, the particle separation test was carry out, the specific gravity of sand is 2.66 , and the maximum and minimum dry density are $1.69 \mathrm{~g} / \mathrm{cm}^{3}$, and $1.30 \mathrm{~g} / \mathrm{cm}^{3}$, respectively. The nonuniform coefficient is 1.91 , and curvature coefficient is 0.89 , which indicated that it belongs to poorly graded medium sand. During the experiment, the fiber is HG-OX2 mortar polypropylene fiber produced by Jiangsu Yancheng henggu Fiber Co., Ltd. The fiber type is round section, bundle monofilament and good dispersion. The linear density, diameter, unit weight, tensile strength, and elasticity modulus of fiber is $5.6 \mathrm{dtex}, 0.025 \mathrm{~mm}, 0.91 \mathrm{~g} / \mathrm{cm}^{3}, 640$ $\mathrm{MPa}$, and $5870 \mathrm{MPa}$, respectively.

In order to ensure the uniform distribution of fibers in soil samples, the amount of samples that may be required for each working condition is estimated in advance, and the required samples are prepared to ensure the consistency of samples. Through repeated tests, it is found that when the water content of sand sample is $5-7 \%$, the fiber is easy to disperse in the mixing process, and is not easy to absorb water and form strips. The specific preparation process as following:

(1) Take a certain amount of sand after passing the $0.075 \mathrm{~mm}$ sieve, subsequently add water and mixing it to make a sand sample with water content of $7 \%$.

(2) According to the set fiber content, the corresponding quality of fiber is weighed, and a small amount of fiber is dispersed in the sand sample. After full stirring, the fiber distribution is relatively uniform. The fiber content can be calculated by

$$
\xi=\frac{m_{f}}{m_{s}} \times 100 \%
$$

Where $\zeta$ is fiber content $(\%) ; m_{\mathrm{f}}$ is fiber mass $(\mathrm{kg}) ; m_{\mathrm{s}}$ is sand mass $(\mathrm{kg})$.

(3) The prepared fiber-reinforced sand sample is sealed with plastic film and then put into the sealing box to prevent water loss. Before each test, take a certain amount of fiber-reinforced sand to dry, and measure its water content. According to the reduction of water content, adding appropriate amount of water to ensure that the water content of the sample is $7 \%$ during the test.

\subsection{Test method}

The equipment used in the test is static and dynamic triaxial torsional multifunctional shear apparatus. The sample is a cylinder with a diameter of $61.8 \mathrm{~mm}$ and a height of $125 \mathrm{~mm}$. The fiber-reinforced sand is divided into 5 parts, which are poured into the sample mold in turn. Each layer is compacted to $25 \mathrm{~mm}$, and the layers are scratched to ensure the internal consistency of the sample, and there is no weak surface. During the saturate process, the $\mathrm{CO}_{2}$ is introduced from the bottom of the sample to exhaust the air in the sample. The ventilation rate is about 2 bubbles per second, and the ventilation time is about 40 minutes. Then, the air free water is injected into the sample to discharge the $\mathrm{CO}_{2}$ in the sample, and the water volume is $1400 \mathrm{ml}$. During the consolidation process, the confining pressure of $100 \mathrm{kPa}$ is applied, and the consolidation time is 40 minutes. After consolidation, the dynamic triaxial test is carried out, and the vibration frequency is $0.1 \mathrm{~Hz}$. The dynamic load is determined according to the corresponding cyclic stress ratio, and the strain of $5 \%$ is taken as the end condition of the test.

The dynamic characteristics of fiber sand were studied by the relative density $D_{\mathrm{r}}=0.5$, confining pressure $\sigma_{3}=100 \mathrm{kPa}$, fiber content $\xi=0.2 \%$ and $0.4 \%$, fiber length $l_{\mathrm{f}}=7 \mathrm{~mm}$ and $13 \mathrm{~mm}$, cyclic stress ratio $\gamma_{\mathrm{c}}=0.22,0.24$, and 0.26 . A total of 12 groups of dynamic triaxial tests were carried out, and the frequency is $0.1 \mathrm{~Hz}$ with the sine waveform. The cyclic stress ratio is defined as

$$
\gamma_{\mathrm{c}}=\frac{\sigma_{\mathrm{d}}}{2 p_{0}}
$$

Where $\gamma_{\mathrm{c}}$ is the cyclic stress ratio, $\sigma_{\mathrm{d}}$ is the dynamic strength, and $p_{0}$ is the initial average consolidation pressure.

\section{Results and discussion}

\subsection{Effect of $\zeta$ and $l_{\mathrm{f}}$ on $u$}

Fig. 1 shows the relationship between the ratio of excess pore water pressure $\left(u / u_{\max }\right)$ and vibration number $(N)$ of fiber-reinforced sand. It can be seen that the $u / u_{\max }$ gradually accumulates with the increase of $N$, and the accumulation rate of sand is faster, while that of fiber-reinforced sand is relatively slow, especially when the fiber content is $0.4 \%$. Under the vibration loading, the indirect contact between the fiber and the particle becomes more closer due to the compaction of the sample. The tensile stress produced by the fiber stretching limits the particle deformation, reduces the porosity, and limits the development of excess pore pressure. It can be seen from the Fig. 1(b) that the growth rate of $u / u_{\max }$ decreased due to the existence of fibers, and the $N$ required for liquefaction increases. When the fiber content is kept constant, the longer fiber can effectively inhibit the accumulation of $u$ and reduce the liquefaction potential.

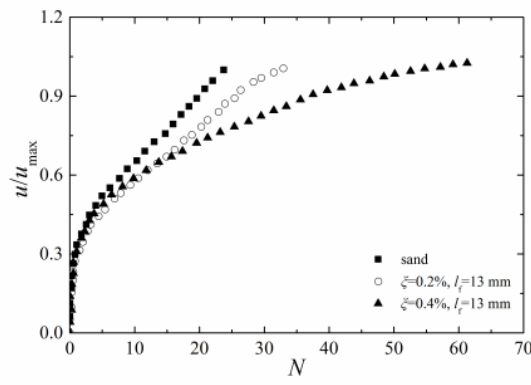

(a) Effect of $\xi$ 


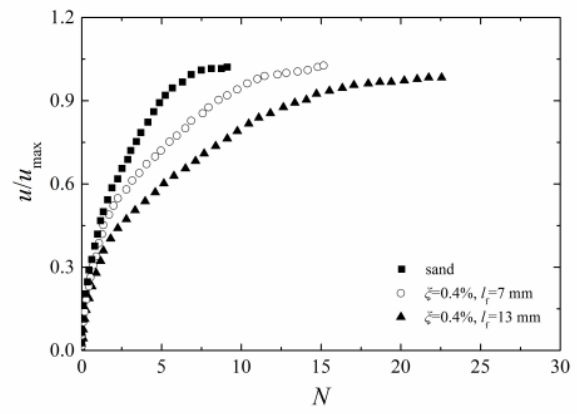

(b) Effect of $l_{\mathrm{f}}$

Fig. 1. Curves of $u / u_{\max }$ versus $N$ under different $\xi$ and $l_{\mathrm{f}}$

\subsection{Effect of $\zeta$ and $I_{\mathrm{f}}$ on $\sigma_{\mathrm{d}}$}

Fig. 2 shows the relationship between $\sigma_{\mathrm{d}}$ and $N$. It can be seen from Fig. 2(a) that when the $\zeta$ is fixed, the $\sigma_{\mathrm{d}}$ of fiber-reinforced sand decreased with the increasing of $N$. Under the same $N$, the $\sigma_{\mathrm{d}}$ of fiber-reinforced sand increased with increasing of $\zeta$. It can be concluded that the $\sigma_{\mathrm{d}}$ of sand can be significantly improved by adding an amount of fibers. It can be seen from Fig. 2(b) that when the $\zeta$ is fixed, the $\sigma_{\mathrm{d}}$ of fiber-reinforced sand with long fiber is higher than that of short fiber, thereby it can be concluded that long fiber is more effective to improve the $\sigma_{\mathrm{d}}$ of fiber-reinforced sand.

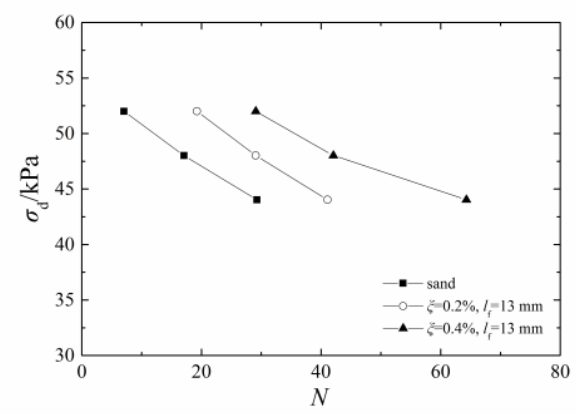

(a) Effect of $\xi$

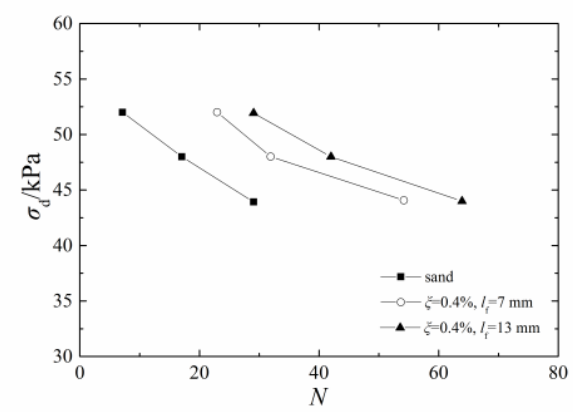

(b) Effect of $l_{\mathrm{f}}$

Fig. 2. Curves of $\sigma_{\mathrm{d}}$ versus $N$ under different $\xi$ and $l_{\mathrm{f}}$

\subsection{Effect of $\zeta$ and $I_{\mathrm{f}}$ on $G_{\mathrm{N}}$}

The dynamic elasticity modulus $\left(E_{\mathrm{d}}\right)$ at a certain time is the average slope of the hysteresis loop, which can be expressed as:

$$
E_{\mathrm{d}}=\frac{\sigma_{\mathrm{d}}}{\varepsilon_{\mathrm{d}}}
$$

Where $\sigma_{\mathrm{d}}$ and $\varepsilon_{\mathrm{d}}$ are the dynamic strength and strain at the vertex of hysteresis loop, respectively.

The dynamic shear modulus $\left(G_{\mathrm{N}}\right)$ corresponding to the $E_{\mathrm{d}}$ can be calculated as:

$$
G_{N}=\frac{E_{\mathrm{d}}}{2(1+v)}
$$

Where $v$ is Poisson's ratio, which taken as 0.5 for saturated loose sand

$G_{\mathrm{N}}$ is an important dynamic parameter to study fiber-reinforced sand, thereby the relationship between $G_{\mathrm{N}} / G_{\max }$ and $N$ was studied under $l_{\mathrm{f}}=13 \mathrm{~mm}$. As shown in Fig. 3, when $l_{\mathrm{f}}=13 \mathrm{~mm}$, with the increase of $N$, the $G_{\mathrm{N}} / G_{\max }$ of fiber-reinforced sand is decreasing, and the rigidity is weakening. Compared with sand, the $G_{\mathrm{N}}$ of fiber-reinforced sand is reduced by a small margin, which indicates that the dynamic properties of fiber-reinforced sand are better than that of sand. When the $\zeta$ is high, its dynamic properties are better than that of low content. When the $\zeta$ is $0.4 \%$, the $G_{\mathrm{N}} / G_{\max }$ of fiber-reinforced sand decreases slowly. It can be seen that when a certain amount of fiber is added to the sand, the stiffness of the fiber-reinforced sand is significantly improved under the dynamic loading. As shown in Fig. 4, when the dynamic loading keep constant, the $G_{\mathrm{N}} / G_{\max }$ of fiber-reinforced sand is slower than that of sand, and that of long fiber is slower than that of short fiber, which indicated that the long fiber has better dynamic characteristics.

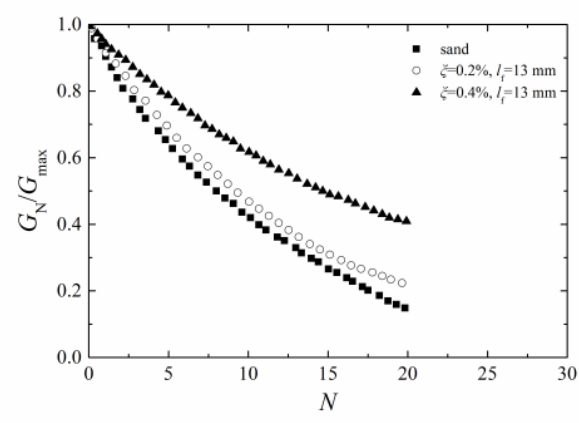

(a) $\sigma_{\mathrm{d}}=44 \mathrm{kPa}$

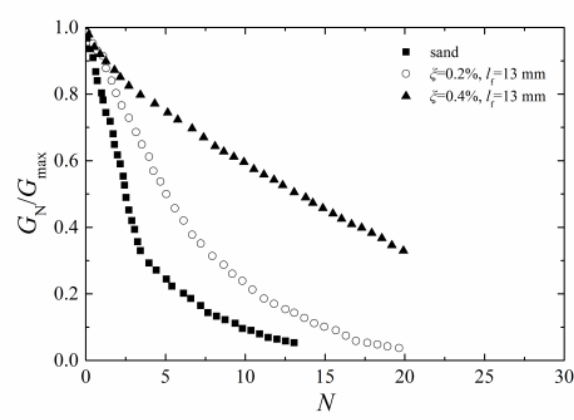

(b) $\sigma_{\mathrm{d}}=48 \mathrm{kPa}$ 


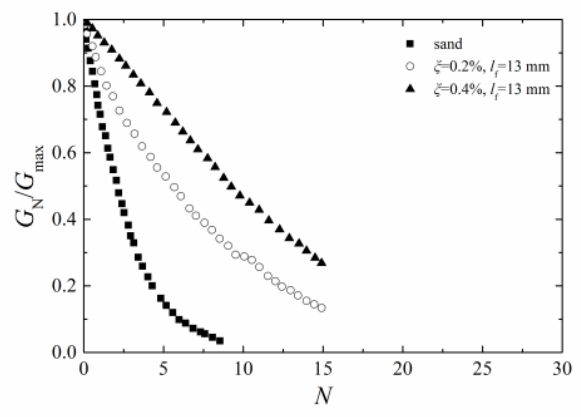

(c) $\sigma_{\mathrm{d}}=52 \mathrm{kPa}$

Fig. 3. Curves of $G_{\mathrm{N}} / G_{\max }$ versus $N$ under different $\xi$

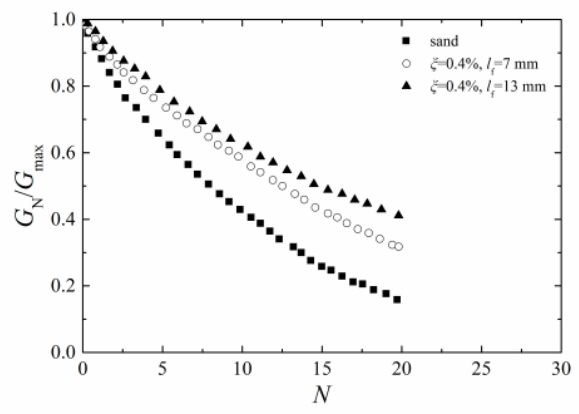

(a) $\sigma_{\mathrm{d}}=44 \mathrm{kPa}$

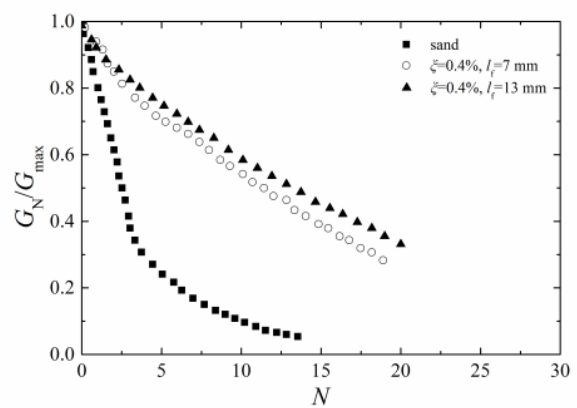

(b) $\sigma_{\mathrm{d}}=48 \mathrm{kPa}$

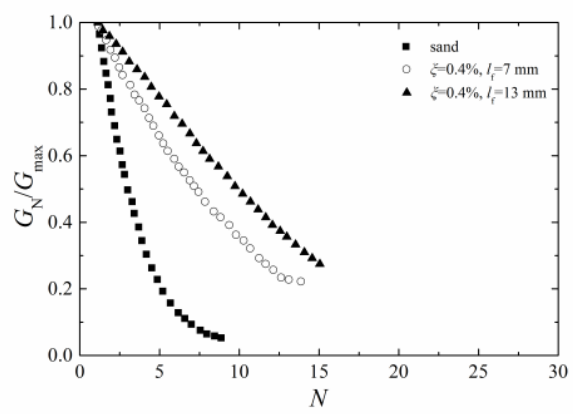

(c) $\sigma_{\mathrm{d}}=52 \mathrm{kPa}$

Fig. 4. Curves of $G_{\mathrm{N}} / G_{\max }$ versus $N$ under different $l_{\mathrm{f}}$

\section{Conclusions}

The dynamic characteristics of fiber-reinforced sand were studied by dynamic triaxial test. The effects of fiber length, fiber content, and cyclic stress ratio on the dynamic response of fiber-reinforced sand were studied. Based on the comprehensive analysis of the test results, the following conclusions can be concluded:
(1) When the fiber length is fixed, the fiber content has a great influence on the dynamic characteristics. With the increasing of fiber content, the dynamic strength increased significantly, the accumulation rate of excess pore pressure decreased, and the liquefaction resistance increased.

(2) When the fiber content is fixed, the fiber length has a great influence on the dynamic characteristics of fiber-reinforced sand. The accumulation rate of excess pore pressure decreased with the increasing of fiber length, and the anti-liquefaction ability is improved. As the fiber length increased, the dynamic shear modulus decreased.

(3) The dynamic properties of fiber-reinforced sand are better than that of sand, and the dynamic strength decreased with the increasing of cyclic stress ratio.

\section{Acknowledgments}

This study was supported by the Special Fund for Scientific Research by Shaanxi Provincial Education Department (18JK1199) and Special Fund for Scientific Research by Xijing University (XJ18T01).

\section{References}

1. K. Ghavami, F.R.D. Toledo, N.P. Barbosa. Behaviour of composite soil reinforced with natural fibers[J]. Cement and Concrete Composites, 21(1): 39-48. (1999)

2. A.L. Welker, N. Josten. Interface friction of a geomem-brane with a fiber reinforced soil[C]//Geo-Frontiers Congress, 1-8. (2005)

3. T. Yetimoglu, O. Salbas. A study on shear strength of sands reinforced with randomly distributed discrete fibers[J]. Geotextiles and Geomembranes, 21(2): 103-110. (2003)

4. N.C. Consoli, M.D.T. Casagrande, P.D.M. Prietto, A. Thome. Plate load test on fiber-reinforced soil[J]. Journal of Geotechnical and Geoenvironmental Engineering, 129(10): 951-955. (2003)

5. M.A.A. Mesbah, J.C. Morel, P. Walker, K. Ghavami. Development of a direct tensile test for compacted earth blocks reinforced with natural fibers[J]. Journal of Materials in Civil Engineering, 16(1): 95-98. (2004)

6. A. Kumar, B.S. Walia, J. Mohan. Compressive strength of fiber reinforced highly compressible clay[J]. Construction and Building Materials, 20(10): 1063-1068. (2006)

7. N.C. Consoli, M.A.A. Bassani, L. Festugato. Effect of fiber-reinforcement on the strength of cemented soils[J]. Geotextiles and Geomembranes, 28(4): 344-351. (2010)

8. S. Akbulut, S. Arasan, E. Kalkan. Modification of clayey soils using scrap tire rubber and synthetic fibers[J]. Applied Clay Science, 38(1-2): 23-32. (2007)

9. A. Hamidi, M. Hooresfand. Effect of fiber reinforcement on triaxial shear behavior of cement treated sand[J]. Geotextiles and Geomembranes, 36: 1-9. (2013)

10. C.S. Tang, B. Shi, W. Gao, F.J. Chen, Y. Cai. Strength and mechanical behavior of short polypropylene fiber reinforced and cement stabilized clayey soil[J]. Geotextiles and Geomembranes, 25(3): 194-202. (2007) 